\title{
A prospective analysis of consultation for difficult urinary catheter insertion at tertiary care centres in Northern Alberta
}

\author{
Chasta Bacsu, MD; Stephan Van Zyl, MD; Keith Francis Rourke, MD, FRCSC
}

Department of Urology, University of Alberta, Edmonton, AB

Cite as: Can Urol Assoc J 2013;7(9-10):343-7. http://dx.doi.org/10.5489/cuaj.574

Published online October 7, 2013.

\section{Abstract}

Background: Difficult urinary catheterization (DUC) is a frequent reason for urologic consultation. Literature regarding DUC is limited. The objective of the study is to examine the current practice pattern of DUC and identify strategies to reduce its incidence and related adverse events.

Methods: This is a prospective observational study of consultation for DUC at tertiary care centres in Edmonton, Alberta between October 2010 and February 2011. All urologic consultations for DUC in adults at the 2 tertiary hospitals were enrolled. Patients were managed according to the current regional standard of care established prior to the study. A clinical encounter questionnaire (CEQ) was completed by the urology service regarding details of the consultation and patient factors. CEQ results were tabulated and analyzed for trends, areas of strengths and weakness in the consultation process.

Results: In total, 89 patients were accrued to the study. Mean age was 67 years and 91\% were male. Seventeen percent of patients had history of previous DUC and 65\% had urologic history. Fortytwo percent of patients had catheter placement without any auxiliary tools. Adverse events, including urosepsis, bladder perforation, hydrouterus, paraphimosis and urethral trauma, were experienced by $37 \%$ of patients. Significant urethral injury as a result of catheterization attempts occurred in 32\%. Forty-one percent of consultations were classified as inappropriate and 53\% occurred between $5 \mathrm{pm}$ and 6:30 am.

Conclusion: DUC is associated with significant patient morbidity and may often be preventable. This study highlights the need for implementation of preventive strategies, widespread education and increased awareness regarding catheter care.

\section{Introduction}

Difficult urinary catheter (DUC) insertion is one of the most common reasons for urological consultation in hospitals. ${ }^{1}$
Despite the prevalence of consultation, literature regarding DUC is extremely limited and focuses predominantly on techniques of successful catheter placement, rather than understanding the clinical situations associated with difficult catheterization consults. Although the incidence of DUC is unknown, many causes have been identified, including anxiety, poor technique, urethral stricture, phimosis, ansarca, bladder neck contracture, false passages, benign prostatic hyperplasia, unfavourable body habitus and patient positioning. ${ }^{1,2}$ DUC can result in significant patient morbidity that in many cases is preventable. Complications of DUC can include urethral abscess, necrotizing infection, rectal injury, urethral stricture, urinary tract infection, urosepsis, hematuria requiring transfusion, as well as extreme physical and emotional distress to the patient. ${ }^{1,3}$ Our study prospectively examines the causes and management of DUC consultation at our institution and secondarily identify areas where focused catheter education could reduce the incidence of DUC and related adverse events.

\section{Methods}

This is an institutional review board-approved, prospective observational study of urologic consultation for DUC at 2 tertiary care centres in Edmonton, Alberta, Canada. The primary objective of the study was to better understand current practice patterns in DUC consultation at our institution. A secondary objective was to identify strategies to reduce the incidence of DUC and related adverse events. All urologic consultations between October 2010 and February 2011 for DUC insertion from 2 tertiary care hospitals in Edmonton were included. Exclusion criteria were patients under 18 years of age, catheter placement performed at elsewhere at another institution or if urological consultation or advice regarding the catheter placement was not needed (i.e., catheters placed by urologic nursing). For the purpose of the study, difficult catheter insertion was defined as a situation where the urological consult service is requested to insert a urinary catheter. 
Bacsu et al.

A 14-item clinical encounter questionnaire (CEQ) was devised to assess pertinent details of the consultation, including demographics of the consulting service, patient factors and degree of difficulty/complexity related to the consultation (Appendix $1 \mathrm{http}: / / j o u r n a l s . s f u . c a / c u a j /$ index.php/journal/article/view/574/1352). The predominant format of the CEQ was multiple choice, with an additional section for comments which permitted analysis by descriptive statistics. Before starting the study, we had an educational session to outline objectives and CEQ format and to ensure the correct completion of the CEQ; this session also acted as recruitment reminders to optimize inclusion of as many DUC consultations as possible. The CEQ was to be completed at the time of each consultation by the urology resident, urology hospitalist or urologist. In a situation where the urinary catheter insertion was anticipated to be difficult, but did not ultimately require a member of the urologic team to place the catheter, a CEQ was completed to ensure an accurate capture of all catheter consultations.

Outcome measures used in this study included number of attempts, timing of consultation in relation to initial catheterization attempts, and additional instrumentation or technique required for catheter insertion by urological service reflecting a more challenging catheterization. Adverse events related to catheter insertion attempts that were assessed include, but not limited to, pain from urinary retention (defined as $>500 \mathrm{~mL}$ postvoid residual urine), new onset gross hematuria after catheter insertion, false urethral passage, traumatic catheter removal, balloon inflation in urethra, or disproportionate patient discontent or pain. There was a separate section for comments to include other adverse events or pertinent information not listed.

The CEQs of all consultations were reviewed at the end of the study period. The investigators reviewed the data for trends, areas of strength and weakness in the present consultation process for DUC insertion to better understand this area and improve patient care.

Sample size goal was 100 consultations over a 20-week period. This allowed an adequate and accurate representation of the usual consultation variety and volume that

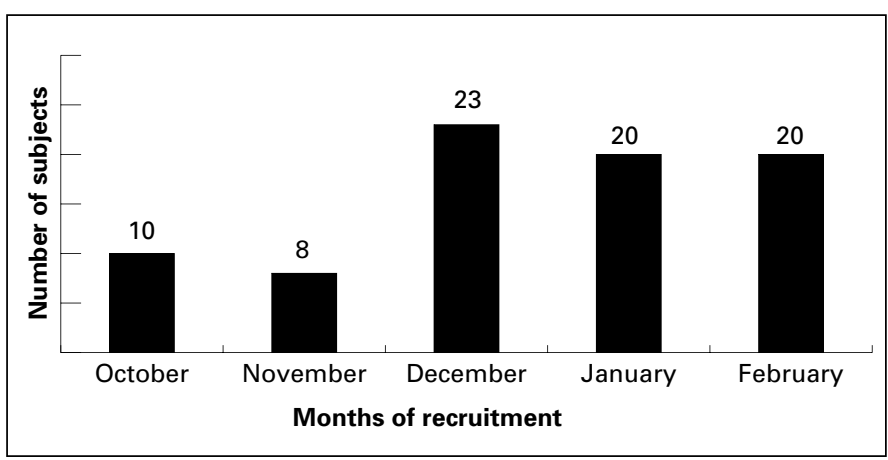

Fig. 1. Number of difficult urinary catheter consults per month during the study period. would be key in understanding our consultation practice. The sample size and duration of the study was calculated based on an estimated pre-study rate of DUC consultation of $6 \pm 2$ per week for the study period at our institution.

\section{Results}

From October 2010 to February 2011, 89 patients were identified, with 81 patients meeting the inclusion criteria. Eight patients were excluded because the catheterization was performed off-site by urology. There was nearly an equal distribution of DUC consultations between each site, with 39 (48\%) at the Royal Alexandra Hospital (RAH) and 42 (52\%) at University of Alberta Hospital (UAH). The frequency of DUC consultations over the study period is represented in Fig. 1. Seventy-four (91\%) DUC consultations occurred in male patients and the mean age at time of DUC consultation was $67 \pm 18$ years. Fourteen patients (17\%) had a history of difficult catheterization and $53(65 \%)$ patients had a significant past urologic history.

\section{Timing, location and initiation of consults}

When assessing the timing of catheter consultation, only $47 \%$ of DUC consultations occurred during "typical" urologic work hours defined as 6:30 to 17:00 hours. The remaining $53 \%$ of consultations occurred outside this time frame, with $36 \%$ occurring between 17:01 and 00:00 hours and 17\% occurring between 00:01 and 6:29 hours. Most DUC consultations $(51 \%)$ were initiated in the emergency department. Other DUC consultations were from inpatient wards (29\%), critical care $(11 \%)$ and operating room $(9 \%)$.

When further analyzing the emergency department DUC consultations, of all the patients included in our study, we found that $37 \%$ were direct to the urologic service from peripheral hospitals outside Edmonton (on-call urologic services were not available at these sites). Moreover, 28\% of DUC consultations were direct transfers from hospitals within Edmonton, with the remaining 35\% of consults initiated by the respective emergency department.

Interestingly, only $51 \%$ of consultations were initiated by a physician with the remaining consultations initiated by nursing $(46 \%)$ or unit clerks (3\%). Referring physicians attempted catheter insertion in less than half $(46 \%)$ of patients with DUC consultations. In $6 \%$ of DUC consultations, no healthcare provider felt comfortable in attempting to place the catheter prior to calling urology.

\section{Indications for difficult urinary catheterization consultation}

The most common reason for catheterization included urinary retention $(40 \%)$, monitoring of urinary output $(30 \%)$, hematuria (12\%), trauma (5\%) and neurogenic bladder (5\%). 
Of the patients with DUC catheter consultation, $65 \%$ had prior urologic history (Fig. 2). The reasons cited for DUC consultation frequently involved the "missing urethra," including but not limited to the obese patient, atrophic vagina, hypospadias, phimosis and perineal urethrostomy. Time to consultation from first attempt to consultation was recorded with a mean of 262 minutes \pm 600 minutes (range: 0-3 days). The mean number of catheterization attempts was $2.7 \pm 2.7$ (range: $0-20$ ). Of the 37 patients with recorded bladder scans, the mean volume at bladder scan was $705 \pm 301 \mathrm{cc}$, with 31 patients $(84 \%)$ in urinary retention and a bladder scan volume of $>500 \mathrm{cc}$. Gross hematuria was present in $28 \%$ of the patients. After each consultation and CEQ, the urologic team member assessed the consult for the degree of difficulty and appropriateness. Of these consults, $40 \%$ were rated as easy, while $60 \%$ were rated as difficult. Based on the completed CEQs, $41 \%$ of DUC were also rated as inappropriate by the urologic team member at the time of consultation. Examples of inappropriate consultation included confirmation of non-traumatic standard or Coude catheter placement without specialized tools, clinical factors suggesting lack of effort by consulting caregiver, patients with ileoconduit diversion and combative patients.

\section{Methods, techniques of catheter placement}

We assessed the methods and techniques used to place the catheters (Table 1). Catheters were not clinically indicated in $2 \%$ of the DUC consultations, and were placed using the standard technique or Coude catheter in $41 \%$. This suggests that up to $43 \%$ of DUC consultations were potentially avoidable. Cystoscopic catheter placement was required in $37 \%$ of patients and $2 \%$ of consultations required a suprapubic catheter.

\section{Adverse events}

Adverse events present at the time of consultation and presumably related to prior catheterization attempts were identified in $37 \%$ of patients (Table 2). Overall, $32 \%$ had evidence of an iatrogenic urethral injury and $9 \%$ had uro-

\begin{tabular}{lc}
\hline $\begin{array}{l}\text { Table 1. Techniques required to successfully place urinary } \\
\text { catheter }\end{array}$ \\
\hline Foley not required & $2(2 \%)$ \\
Standard insertion & $15(19 \%)$ \\
Coudé catheter & $17(21 \%)$ \\
Guidewire or catheter & $15(19 \%)$ \\
guide & $30(37 \%)$ \\
Cystoscopy & $(17 \%)$ \\
$\quad$ + Guidewire alone & $(20 \%)$ \\
$\quad$ Dilators & $2(2 \%)$ \\
Suprapubic catheter &
\end{tabular}

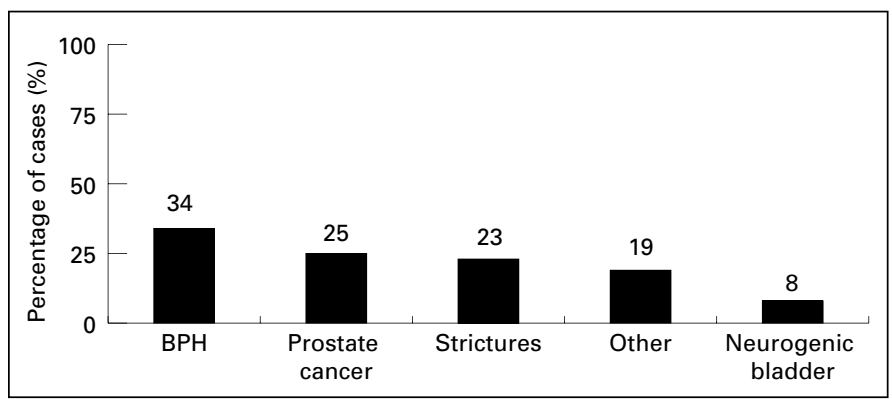

Fig. 2. Reasons for difficult urinary catheter consultation.

sepsis at the time of consultation. Other adverse events in this series included paraphimosis, bladder perforation and hydrouterus as a result of continuous bladder irrigation mistakenly placed in the vagina for 3 days while the patient suffered in clot retention.

\section{Discussion}

Although DUC consultation is a common part of urologic practice and likely results in negative patient experience and potential adverse events, the medical literature regarding this topic is very limited. This paucity of literature may undermine the true clinical significance of DUC. Our study demonstrates a higher number of DUC consults and a higher incidence of ancillary tools required for placement of DUC compared to a recent abstract. ${ }^{4}$ This Stanford-based study cited only 47 consults for catheter placement at 4 different teaching hospitals during an 8-month study period, with 70\% of catheters placed using the standard technique or Coude catheter. ${ }^{4}$ Our current study had almost twice as many DUC consultations despite including fewer hospitals in a shorter study period. Only $41 \%$ of consultations in our series could be managed by standard or Coude catheter placement without ancillary tools. We did not include cases where urology nurses were contacted and successfully managed the DUC rather than the urologic consult team. If catheters placed by urologic nurses were included, it is anticipated that there would be an increased overall number of DUC events and a greater percentage of patients with easy-to-place catheters. If urologic nurses tried but was unable to place the Coude,

Table 2. Adverse events with DUC

\begin{tabular}{lcc}
\hline Total no. patients with adverse events & 30 & $(37 \%)$ \\
Total no. adverse events & 37 & \\
False passage & 26 & $(32 \%)$ \\
Urosepsis & 7 & $(9 \%)$ \\
UTI without sepsis & 1 & $(1 \%)$ \\
Bladder perforation & 1 & $(1 \%)$ \\
Paraphimosis & 1 & $(1 \%)$ \\
Hydrouterus & 1 & $(1 \%)$ \\
\hline UTI: urinary tract infection. & \multicolumn{3}{l}{} \\
\hline
\end{tabular}


Bacsu et al.

3-way or standard catheter, the DUC consults were initiated and included in the study as it was thought to be a more complex situation justifying of consultation. The high consultation rate in our study may be attributed to the fact that $49 \%$ of consultations were initiated by a non-physician and $46 \%$ had a physician-attempted catheter placement. The lack of physician involvement in the catheterization process prior to DUC consult at our institution may reflect physician inexperience, apathy or lack of education in proper catheter insertion technique. Routine urinary catheter insertion should be part of every practicing physician's basic clinical skill set and not included as a sub-specialty procedure. The alarming fact that $6 \%$ of consultations are made in which not a single healthcare provider even attempted catheter placement may emphasize that some healthcare professionals feel unqualified, overwhelmed or inexperienced with catheter insertion.

Our study also identified a delay in DUC consultation from the time of first catheter attempt. The mean time to consultation from first attempt was 262 minutes, with a mode of 120 minutes and range of 0 to 3 days. This delay can cause substantial patient anxiety and discomfort and may predispose patients to significant complications, such as urosepsis, periurethral abscess or other infectious complications.

Additionally, more than half $(53 \%)$ of consultations occurred outside normal work hours (defined as 6:30 to 17:00). This timing of consultation could indicate a lack of awareness at identifying patients at risk for DUC. This could be improved if at-risk patients could be recognized earlier in their clinical course. One unfortunate patient had 20 attempts at catheterization prior to initiation of urologic consultation. This patient not surprisingly suffered a significant iatrogenic urethral injury. There appears to be a pronounced spectrum of healthcare providers with some level of fear in attempting a routine urinary catheterization, while others are willing to attempt extreme measures to avoid urologic consultation.

Our study also demonstrated a higher rate of catheterinsertion adverse events (37\%) than currently reported. During a 6-month term, Kasefi and colleagues found that were 14 consultations for catheter insertion-related adverse events (a calculated rate of incidence of 3.2/1000 patients); this was decreased to 3 consultations in the following 6-month term after the implementation of a catheter education program to reduce the calculated incidence to $0.7 / 1000$ patients. ${ }^{2}$ The most severe complications in our study was a bladder perforation from a catheter guide and a 3-way catheter placed vaginally with continuous bladder irrigation resulting in hydrouterus and clot retention of the urinary bladder. Paraphimosis did occur, but no cases required emergent surgical treatment. The incidence of longterm catheter complications, such as urethral stricture, was not assessed by our study and this may under-represent this incidence of adverse events. The long-term sequelae of DUC and associated iatrogenic injuries likely warrant further investigation. The high rate of adverse events related to DUC in our series was surprising and demonstrates an area of patient care that demands substantial improvement. A catheter education program appears to be an effective strategy at reducing catheter-related complication. ${ }^{2}$ We feel that multifaceted catheter education models should also be emphasized in both medical school and residency training as routine urinary catheter placement should be part of a physician's basic clinical skill set. In addition, multifaceted educational programs to all disciples outlining specific indications for urinary catheterization can significantly reduce the incidence of patients inappropriately receiving an indwelling urinary catheter. This in turn would reduce the amount of unnecessary urethral trauma and incidental DUC consultations. ${ }^{5}$

The economic and health-related costs of DUC and related adverse events are not known and have potential for further study. In this series, $28 \%$ were transferred from another hospital within the Edmonton and another $37 \%$ were transferred from outside of Edmonton, most of which required emergency ambulance transportation for catheter insertion.

The prospective nature of the study with completion of CEQs is a unique approach to assess DUC consultations. However, it was not possible to determine the exact incidence of DUC consultation in both hospitals. The study also relied on the participation of all members of the urology consulting team and that could be perceived as a potential limitation if certain team members did not routinely complete the CEQs. This could potentially underestimate this already prevalent clinical problem. However, acceptance of the CEQ for DUC consultations was well-received and this analysis is believed to accurately reflect the consultation pattern at our institution.

\section{Conclusion}

DUC is associated with significant patient morbidity and may often be preventable. The magnitude of DUC consultations and associated adverse outcomes in our series highlights the need for implementation of preventive strategies, widespread education and increased awareness regarding catheter care.

Competing interests: None declared.

This paper has been peer-reviewed. 


\section{References}

1. Villanueva $C$, Hemstreet GP. Difficult male urethral catheterization: A review of different approaches. Int Braz J Urol 2008;34:401-22. http://dx.doi.org/10.1590/S1677-55382008000400002

2. Kashefi B, Messer K, Barden R, et al. Incidence and prevalence of iatrogenic urethral injury. I Urol 2008;179:2254-8. http://dx.doi.org/10.1016/i.juro.2008.01.108

3. Leuck AM, Wright D, Ellingson L. Complications of Foley catheters-is infection the greatest risk? I Urol 2012;187:1662-6. http://dx.doi.org/10.1016/i.juro.2011.12.113
4. Liu J-J, Kouba EJ, Thong A, et al. Patterns of Catheterization Consults in a Teaching Institution (Abstract 293). Presented at WS AUA 2010, Hawaii, 2010 0ct 24-29.

5. Knoll $B M$, Wright $D$, Ellingson $L$, et al. Reduction of inappropriate urinary catheter use at a Veterans Affairs hospital through a multifaceted quality improvement project. Clin Infect Dis 2011;52:1283-90. http:// dx.doi.org/10.1093/cid/cir188

Correspondence: Dr. Keith Francis Rourke, Hys Medical Centre, Suite 400 - 11010 101st Street NW, Edmonton, $A B$ 